\title{
A NOTE ON POWER BOUNDED MATRICES*
}

\author{
R.E. HARTWIG ${ }^{\dagger}$ AND P. PATRICIO ${ }^{\ddagger}$
}

\begin{abstract}
The concept of focal power is used to examine when a nonnegative power bounded matrix is periodic.
\end{abstract}

Key words. Power bounded, Focal power, Nonnegative.

AMS subject classifications. 15A21, 15A48, 15A57.

1. Introduction. A complex $n \times n$ matrix is power bounded - shortened to $\mathrm{PB}$ - when there is a nonnegative $M$ such that the inequalities $\left|\left(A^{k}\right)_{i j}\right| \leq M$ hold for all $i, j \in\{1, \ldots, n\}$ and $k \in \mathbb{N}$.

A special subclass are the periodic matrices $A$, for which $A^{k+h}=A^{k}$ for some nonnegative integers $h$ and $k$.

Given a block matrix $A=\left[\begin{array}{cc}A_{11} & A_{12} \\ A_{21} & A_{22}\end{array}\right]$ with $A_{11}$ square, we say it is $(1,1)$ focused (as defined in [7]) if $A^{k}=\left[\begin{array}{cc}A_{11}^{k} & ? \\ ? & ?\end{array}\right]$ for all $k$, in which case $A$ is said to have focal power.

We say $A$ is convergent to the matrix $X=\left[x_{i j}\right]$ if the sequences $\left(A^{k}\right)_{i j}$ converge to $x_{i j}$ as $k \rightarrow \infty$. A matrix is convergent if it is convergent to some matrix. This is a special case of convergence as defined in [4, Definition 10.1], for the matrix sequence $\left\{A, A^{2}, A^{3}, \ldots\right\}$. In particular, we say $A$ is zero convergent when $A$ is convergent to the zero matrix. We distinguish between convergence and zero convergence (i.e., convergence to the zero matrix) of $A$. Needless to say, $A$ can be power bounded without being convergent, as seen from the example $A=-I$.

We denote the rank, the range and the nullspace of a matrix $A$ by $\operatorname{rank}(A)$, $R(A)$ and $N(A)$ respectively. The spectrum and the spectral radius of $A$ are denoted, respectively, by $\sigma(A)$ and $\rho(A)$. For $\lambda \in \sigma(A)$, its algebraic multiplicity is denoted by

* Received by the editors on October 12, 2008. Accepted for publication on May 26, 2012. Handling Editor: Roger A. Horn.

${ }^{\dagger}$ Mathematics Department, NCSU Raleigh, NC 27695-8205, USA (hartwig@unity.ncsu.edu).

${ }^{\ddagger}$ Departamento de Matemática e Aplicações, Universidade do Minho, 4710-057 Braga, Portugal (pedro@math.uminho.pt). Member of the research unit CMAT - Centro de Matemática da Universidade do Minho, Portugal. 
$n(\lambda)$ and its index by $i n(\lambda)$. The index $i n(A)$ of $A$ is defined as $i n(0)$ and permutation similarity is denoted by $\underset{\text { per }}{\approx}$.

Given a matrix $A$ with spectrum $\sigma(A)=\left\{\lambda_{1}, \ldots, \lambda_{s}\right\}$, we define the spectral components of $A$ as $Z_{k}^{j}=\left(A-\lambda_{k} I\right)^{j}\left(I-\left(A-\lambda_{k} I\right)\left(A-\lambda_{k} I\right)^{D}\right) \frac{1}{j !}$, where $X^{D}$ denotes the Drazin inverse of $X$ (see [3]). When $i n(A)=1, X^{D}$ becomes the group inverse of $X$ and is denoted by $X^{\#}$. In particular, when $j=0, Z_{k}^{0}(A)=I-\left(A-\lambda_{k} I\right)\left(A-\lambda_{k} I\right)^{D}$.

The question of when a matrix is PB is an old one, and has been examined by numerous authors, e.g., [9], 11], 10, [12, 13]. Likewise, periodic matrices have also been investigated, as for example in [6]. On the other hand, focal power has only recently been introduced [7, and appears naturally in the study of PB matrices.

In this paper, we first address the general complex case with aid of the Jordan form and its spectral properties. In addition a special canonical form is used. We subsequently derive results for block triangular matrices, and lastly, consider nonnegative matrices.

For a real nonnegative matrix $A$, we may combine the Frobenius normal form (FNF), or the irreducible normal form [8, page 506], with the critical normal form (see Section 4) to obtain a block upper triangular form for nonnegative PB matrices. We give a direct proof that avoids minimal polynomials or generalized eigenvectors. An alternative approach is to use the structure of the diagonal entries in the matrix, which is addressed at the end of this paper.

When $A$ is nilpotent, then $A$ is clearly periodic and PB. Recalling that a nilpotent matrix is characterized by the fact that all its powers have zero trace, we may conclude that if $A$ is not nilpotent then there exists a power of $A$ with nonzero trace. Moreover when, in addition, $A$ is nonnegative then it has a FNF of the type

$$
F_{A}=\left[\begin{array}{ccc}
0 & ? & ? \\
0 & 0 & \\
& & \ddots \\
0 & & 0
\end{array}\right]
$$

An important matrix in applied matrix theory is the basic circulant matrix

$$
\Omega=\left[\begin{array}{ccccc}
0 & & \cdots & & 1 \\
1 & 0 & & & \\
0 & 1 & 0 & & \vdots \\
& & \ddots & \ddots & \\
0 & & & 1 & 0
\end{array}\right]_{n \times n} .
$$


It is nonnegative, irreducible and bounded, but it is not convergent. It is periodic because $\Omega^{n}=I$.

For a nonnegative matrix $A$ with $\rho(A) \leq 1$, the existence of positive right or left eigenvectors associated with the Perron root is not a necessary condition for a nonnegative matrix to be $\mathrm{PB}$, as seen from the following example.

Example 1.1. The matrix $A=\left[\begin{array}{ll}1 & 0 \\ 0 & \frac{1}{2}\end{array}\right]$ with $\rho(A)=1$, shows that $A$ can be convergent (and thus, be PB) without having a positive left or right (Perron) eigenvector. Indeed, $\left[\begin{array}{l}1 \\ 0\end{array}\right]$ is a right and left eigenvector of $A$ associated with the eigenvalue $\rho(A)=1$.

As a special case, we examine nonnegative $\mathrm{PB}$ matrices whose powers have diagonal entries that are 0 or 1 exclusively. We use the concept of $(1,1)$ Focal Power to show that these matrices must be periodic. Indeed, some power must be idempotent. This simplifies and extends in part some of the results of Yang and Zhang [14].

The stochastic matrix $P=\left[\begin{array}{c|cc}1 & 0 & 0 \\ \hline \frac{1}{2} & 0 & \frac{1}{2} \\ \frac{1}{2} & \frac{1}{2} & 0\end{array}\right]$ shows that for a matrix to be periodic, it does not suffice for it to be PB, nonnegative, with 0-1 diagonal entries. Indeed, because $P^{3}=P^{2}+(1 / 4) P-(1 / 4) I, P$ is not periodic.

2. General results. For a square matrix $A$, we define its geometric and Cesaro sums respectively by

$$
G_{N}(A)=I+A+\cdots+A^{N-1} \text { and } \mathcal{C}_{\mathcal{N}}(\mathcal{A})=\frac{1}{N} G_{N}(A)
$$

The Jordan form can be used to establish the following theorem.

Theorem 2.1. Let $A$ be a square complex matrix and $\lambda \in \sigma(A)$.

1. The following are equivalent, as $N \rightarrow \infty$ :

(a) $A^{N}$ is bounded;

(b) $A^{N} / N \rightarrow 0$;

(c) for $\lambda \in \sigma(A),|\lambda| \leq 1$ and $|\lambda|=1 \Rightarrow \operatorname{in}(\lambda)=1$;

(d) The Cesaro sums $\mathcal{C}_{N}(A)$ converge.

2. The following are equivalent, as $N \rightarrow \infty$ :

(a) $A^{N} \rightarrow 0$;

(b) The geometric sums $G_{N}(A)$ converge;

(c) $\rho(A)<1$.

3. The following are equivalent, as $N \rightarrow \infty$ : 
(a) $A^{N}$ is bounded and $1 \notin \sigma(A)$;

(b) $G_{N}(A)$ is bounded;

(c) $\mathcal{C}_{N}(A) \rightarrow 0$;

(d) $|\lambda| \leq 1, \lambda \neq 1$ and $|\lambda|=1 \Rightarrow \operatorname{in}(\lambda)=1$.

4. The following are equivalent:

(a) A is convergent;

(b) $|\lambda| \leq 1$ and $|\lambda|=1 \Rightarrow \lambda=1$ with $\operatorname{in}(\lambda)=1$.

We next recall ([3, Lemma 1.2]) that if $A$ is a matrix with minimal polynomial $\psi_{A}(\lambda)=(\lambda-1)^{s} f(\lambda)$ such that $\operatorname{gcd}(\lambda-1, f)=1$, then

$$
G_{N}(A)=\sum_{i=0}^{N-1} A^{i}=(I-A)^{D}\left(I-A^{N}\right)+\sum_{i=0}^{s-1}\left(\begin{array}{c}
N \\
i+1
\end{array}\right)(A-I)^{i} Z_{1}^{0}
$$

Now suppose $A$ is $\mathrm{PB}$, which ensures that the group inverse $(I-A)^{\#}$ exists (see [1).

If $1 \notin \sigma(A)$ then $I-A$ has an inverse and

$$
(I-A) \sum_{i=0}^{N-1} A^{i}=I-A^{N} \Rightarrow G_{N}=(I-A)^{-1}\left(I-A^{N}\right) .
$$

On the other hand, if $1 \in \sigma(A)$ then we have $m_{1}=i n(1)=1$, ie, $\psi_{A}(\lambda)=(\lambda-1) f(\lambda)$ with $f(1) \neq 0$, which gives

$G_{N}(A)=(I-A)^{\#}\left(I-A^{N}\right)+N Z_{1}^{0}=(I-A)^{\#}\left(I-A^{N}\right)+N\left[I-(I-A)(I-A)^{\#}\right]$.

Another tool to study PB matrices is the RCNZ (row-column-nonzero) canonical form

$$
A=\left[\begin{array}{cccc}
P & Q & 0 & 0 \\
0 & 0 & 0 & 0 \\
R & S & 0 & 0 \\
0 & 0 & 0 & 0
\end{array}\right]
$$

where $\left[\begin{array}{ll}P & Q \\ R & S\end{array}\right]$ has no zero row or column. It is easily seen that

$$
A^{k}=\left[\begin{array}{cccc}
P^{k} & P^{k-1} Q & 0 & 0 \\
0 & 0 & 0 & 0 \\
R P^{k-1} & R P^{k-2} Q & 0 & 0 \\
0 & 0 & 0 & 0
\end{array}\right]
$$

and hence, that $A$ and $P$ have a similar behavior, namely, 
(i) $A^{N}$ is (zero) convergent if and only if $P^{N}$ is (zero) convergent,

(ii) $A$ is $\mathrm{PB}$ if and only if $P$ is $\mathrm{PB}$,

(iii) $A^{N} / N \longrightarrow 0$ if and only if $P^{N} / N \longrightarrow 0$,

(iv) $G_{N}(A)$ is bounded if and only if $G_{N}(P)$ is bounded,

(v) $\operatorname{Tr}\left(A^{k}\right)=\operatorname{Tr}\left(P^{k}\right)$ etc.

Moreover, $A$ is periodic, say $A^{k}=A^{k+h}$ for some nonnegative integers $h$ and $k$, if and only if $P^{k}=P^{k+h}, P^{k-1} Q=P^{k-1+h} Q$, and $R P^{k}=R P^{k-1+h}$.

3. The block triangular case. Before we turn to the general nonnegative case let us give a few preliminary results dealing with block triangular matrices.

Lemma 3.1. Let $M=\left[\begin{array}{ccc}A & ? & C \\ 0 & X & ? \\ 0 & 0 & D\end{array}\right]$ with $\rho(A)<1$ and $\rho(D)<1$. Then, $M$ is $P B$ if and only if $X$ is $P B$.

Proof. The necessity is clear, so suppose that $X$ is PB. Then its minimal polynomial is given by $\psi_{X}(x)=f(x) g(x)$, where $f$ only has distinct roots on the unit circle and all the roots of $g$ are inside the unit circle. Now because

$$
\operatorname{Lcm}\left(\psi_{A}, \psi_{X}, \psi_{D}\right)\left|\psi_{M}\right| \psi_{A} \cdot \psi_{X} \cdot \psi_{D}
$$

we see that $f\left|\psi_{M}\right| f \cdot g \cdot \psi_{A} \cdot \psi_{D}$, and hence, $\psi_{M}=f(x) \cdot h(x)$, where all the roots of $h$ lie inside the unit circle. This means that $M$ has inherited the property that all eigenvalues on the unit circle have index 1 , as desired.

Let us start with the simplest case where $M=\left[\begin{array}{cc}A & C \\ 0 & D\end{array}\right]$. It follows by induction that $M^{N}=\left[\begin{array}{cc}A^{N} & \Gamma_{N} \\ 0 & D^{N}\end{array}\right]$, where

$$
\Gamma_{N}(A, C, D)=\sum_{k=0}^{N-1} A^{N-k-1} C D^{k}=A \Gamma_{N-1}+C D^{N-1}
$$

We note that $M$ is $\mathrm{PB}$ if and only if $A$ and $D$ are $\mathrm{PB}$ and $\Gamma_{N}$ is bounded for all $N$. In particular, Theorem 2.1 ensures that, for each $\lambda_{A} \in \sigma(A), \lambda_{D} \in \sigma(D)$, we have $\left|\lambda_{A}\right| \leq 1,\left|\lambda_{D}\right| \leq 1$ with index at most one on the Perron circle $|\lambda|=1$.

If in addition $\mathbf{u}^{T} A=\alpha \mathbf{u}^{T}$ and $D \mathbf{v}=\delta \mathbf{v}$ with $\alpha, \delta \neq 0$, then $\mathbf{u}^{T} \Gamma_{N} \mathbf{v}=$ $\left(\mathbf{u}^{T} C \mathbf{v}\right) S_{N}$, where

$$
S_{N}=\sum_{k=0}^{N-1} \alpha^{N-k-1} \delta^{k}=\left\{\begin{array}{cc}
N \alpha^{N-1} & \text { if } \alpha=\delta \\
\frac{\alpha^{N}-\delta^{N}}{\alpha-\delta} & \text { if } \alpha \neq \delta
\end{array}\right.
$$


LEMma 3.2. $S_{N}$ is bounded for all $N$ if and only if either $\alpha=\delta$ with $|\alpha|<1$ or $\alpha \neq \delta$ with $|\alpha| \leq 1,|\delta| \leq 1$.

We may therefore state that:

if $\Gamma_{N}$ is bounded for all $N$ then $\mathbf{u}^{T} \Gamma_{N} \mathbf{v}=\left(\mathbf{u}^{T} C \mathbf{v}\right) S_{N}$ is bounded for all $N$.

This leads to

Corollary 3.3. $\left(\mathbf{u}^{T} C \mathbf{v}\right) S_{N}$ is bounded for all $N$ if and only if either $\mathbf{u}^{T} C \mathbf{v}=0$ with $S_{N}$ arbitrary, or $\mathbf{u}^{T} C \mathbf{v} \neq 0$ and $S_{N}$ is bounded for all $N$.

For the general block triangular case, suppose that

$$
M=\left[\begin{array}{c|ccc|c}
A & & X_{1} & & C_{1} \\
\hline 0 & ? & & ? & \\
0 & 0 & ? & & Y_{1} \\
0 & 0 & & ? & \\
\hline 0 & 0 & \cdots & 0 & D
\end{array}\right]
$$

and $\quad M^{N}=\left[\begin{array}{c|ccc|c}A^{N} & & X_{N} & & C_{N} \\ \hline 0 & ? & & ? & \\ 0 & 0 & ? & & Y_{N} \\ 0 & 0 & & ? & \\ \hline 0 & 0 & \cdots & 0 & D^{N}\end{array}\right]$.

We shall show by induction that $C_{N}=\Gamma_{N}(A, C, D)+Z_{N}$ for some $Z_{N}$. Indeed, first observe that it holds for $N=2$ with $Z_{2}=0$. Now assuming it holds for $N$, we see that $C_{N+1}=A C_{N}+X_{1} Y_{N}+C D^{N}=\left(A \Gamma_{N}+C D^{N}\right)+\left(A Z_{N}+X_{1} Y_{N}\right)=$ $\Gamma_{N+1}(A, C, D)+Z_{N+1}$.

We may now draw several easy conclusions for nonnegative matrices.

- Since $Z_{N} \geq 0$ we have

$$
C_{N} \geq \Gamma_{N}(A, C, D)=\sum_{k=0}^{N-1} A^{N-k-1} C D^{k} .
$$

- If $\mathbf{u}>\mathbf{0}, \mathbf{v}>\mathbf{0}$ and $\Gamma_{N} \geq 0$, then $\mathbf{u}^{T} \Gamma_{N} \mathbf{v}=0$ if and only if $\Gamma_{N}=0$.

- If we fix $k \geq 1$, then by applying (3.1) to $\left(M^{k}\right)^{N}$ we see that

$$
C_{N^{k}} \geq \Gamma_{N}\left(A^{k}, C_{k}, D^{k}\right), \text { for } k=1,2,3, \ldots
$$

- If $M$ is $\mathrm{PB}$ so is $M^{k}$, and hence, $C_{N^{k}}$ and $\Gamma_{N}\left(A^{k}, C_{k}, D^{k}\right)$ are bounded for all $N$.

This yields

Theorem 3.4. Suppose $M=\left[\begin{array}{cc}A & C \\ 0 & D\end{array}\right] \geq 0$ and $M$ is $P B$, with $\mathbf{u}^{T} A=\mathbf{u}^{T}$ and $D \mathbf{v}=\mathbf{v}$, where $\mathbf{u}>\mathbf{0}, \mathbf{v}>\mathbf{0}$. Then

(i) $M^{k}$ is $P B$ and $\mathbf{u}^{T} A^{k}=\mathbf{u}^{T}, D^{k} \mathbf{v}=\mathbf{v}$ for $k=1,2, \ldots$, and 
(ii) $C_{k}=0$.

Proof. Condition (ii) follows from the fact that we may apply Corollary 3.3 to the matrices $M^{k}, A^{k}$, and $D^{k}$. Indeed, since $\alpha^{k}=\delta^{k}=1, S_{N^{k}}$ cannot be bounded and we must have $\mathbf{u}^{T} C_{k} \mathbf{v}=0$, which ensures that $C_{k}=0$.

4. The Frobenius and critical normal forms. Let us now turn to the Frobenius normal form (FNF) of our matrix $M$. Recall that with a nonnegative matrix $M$ we may associate the digraph $G_{M}=(V, \mathcal{E})$, where $V=\left\{S_{1}, \ldots, S_{n}\right\}$ is a set of nodes (sites), and $\mathcal{E}=\left\{\left(S_{i_{r}}, S_{j_{s}}\right)\right\}$ is the set of arcs (pairs), such that the pair $\left(S_{i}, S_{j}\right) \in \mathcal{E}$. That is, we draw an arc from $S_{i}$ to $S_{j}$ and write $S_{i} \rightarrow S_{j}$, exactly when $m_{i j} \neq 0$. If there is a path from $S_{i}$ to $S_{j}$, we write $S_{i} \longrightarrow S_{j}$ and if there is no path we indicate this by $S_{i} \nrightarrow S_{j}$.

We define the equivalence relation on $\mathrm{V}$, by $S_{i} \sim S_{j}$ if and only if $S_{i}=S_{j}$ or $S_{i} \longrightarrow S_{j}$ and $S_{j} \longrightarrow S_{i}$. The equivalence classes $\left\{V_{1}, \ldots, V_{r}\right\}$ are called the "condensed nodes" of G. The condensed graph $G_{M}^{*}$ of M is obtained by drawing an arc from $V_{p}$ to $V_{q}, p \neq q$, if and only if there is some arc in $G_{M}$ from a node in $V_{p}$ to some node in $V_{q}$. The condensed graph has no cycles and the condensed nodes can be relabeled such that the matrix $M$ is permutation similar to its FNF

$$
F_{M}=\left[\begin{array}{cccccc}
A_{11} & & \cdots & & & ? \\
0 & A_{22} & & & & \\
& & \ddots & & A_{p q} & \\
0 & 0 & 0 & A_{r r} & & ? \\
& & & & \ddots & \\
0 & & & & & A_{w w}
\end{array}\right]\left(A_{i i} \text { irreducible or } 0_{1 \times 1}\right)
$$

Thus, the block $A_{p q}$ of $F_{M}$ is nonzero if and only if there is an $\operatorname{arc}$ from $V_{p}$ to $V_{q}$. For convenience we shall shorten $A_{i i}$ to $A_{i}$.

If $M^{N}$ is bounded, so is $F_{M}^{N}$. Hence we know, for any eigenvalue $\lambda$ of $M$, that $|\lambda| \leq 1$ or $|\lambda|=1$ with $\operatorname{in}(\lambda)=1$.

This means that the diagonal blocks in $F$ can be separated into "singular blocks" with $\rho\left(A_{i}\right)=1$ and the remaining "nonsingular" blocks with $\rho\left(A_{j}\right)<1$. Therefore, no nonsingular block contains any eigenvalue on the unit circle.

Consider two singular blocks $A_{p}^{T}$ and $A_{q}$ with $p<q$ with positive eigenvectors $\mathbf{u}$ and $\mathbf{v}$ associated with their spectral radius. Then the submatrix $M_{p q}=$ 
$\left[\begin{array}{ccc}A_{p} & ? & C_{p q} \\ 0 & ? & ? \\ 0 & 0 & A_{q}\end{array}\right]$ has powers that are also bounded. Moreover, if

$$
M_{p q}^{k}=\left[\begin{array}{ccc}
A_{p}^{k} & ? & C_{p q}^{(k)} \\
0 & ? & ? \\
0 & 0 & A_{q}^{k}
\end{array}\right]
$$

then by Theorem 3.4 we may conclude that $C_{p q}^{(k)}=0$ for all $k=1,2, \ldots$

In terms of the condensed graph of $M$, this means that there is no k-step path in $G_{M}^{*}$ from a singular node $V_{p}$ to any singular node $V_{q}$. Since $k$ is arbitrary, that means that there is no path (no access) from $V_{p}$ to $V_{q}$.

We now permute the FNF further to the "critical normal form", in which we take the "critical nodes" $V_{i}$ to be those nodes for which $\rho\left(A_{i}\right)=1$.

Suppose that

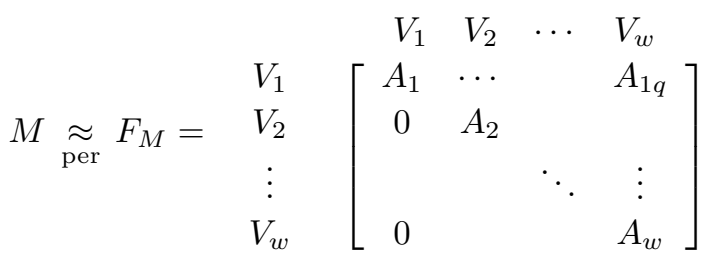

and that $V_{k}$ is the first critical node in the sequence $\left(V_{1}, \ldots, V_{w}\right)$. We now partition $V$ into $V=V_{k} \cup V_{k}^{=} \cup V_{k}^{\neq}$, where $V_{k}^{=}=\left\{V_{i} ; V_{i} \longrightarrow V_{k}\right\}$ contains all the (condensed) nodes that have access to $V_{k}$ and $V_{k}^{\neq}=\left\{V_{i} ; V_{i} \nrightarrow V_{k}\right\}$ is made up of all the nodes that have no access to $V_{k}$. If $U$ and $W$ are two subsets of nodes of the graph (i.e., of $\mathrm{V}$ ), then we write $U \rightarrow W$, if $u \rightarrow w$ for some $u \in U, w \in W$, and $U \nrightarrow \rightarrow W$ otherwise. We now note that:

(i) $V_{k}^{\neq} \not \rightarrow V_{k}^{=}$otherwise if $x \longrightarrow y$ with $x \in V_{k}^{\neq}$and $y \in V_{k}^{=}$, then $x \longrightarrow y \longrightarrow V_{k}$ which cannot be.

(ii) $V_{k} \nrightarrow \rightarrow V_{k}$ otherwise we have a loop in $G^{*}$.

(iii) $V_{k} \in V_{k}^{\neq}$, since otherwise we have a cycle $G^{*}$.

(iv) $V_{k}^{\neq} \nrightarrow V_{k}$ by definition.

(v) $V_{k}=\subseteq\left\{V_{1}, \ldots, V_{k-1}\right\}$ and $\left\{V_{k+1}, \ldots, V_{w}\right\} \subseteq V_{k}^{\neq}$because the later nodes have no access to $V_{k}$. 
If we rearrange the nodes in $V$, keeping the indices in increasing order, we arrive at

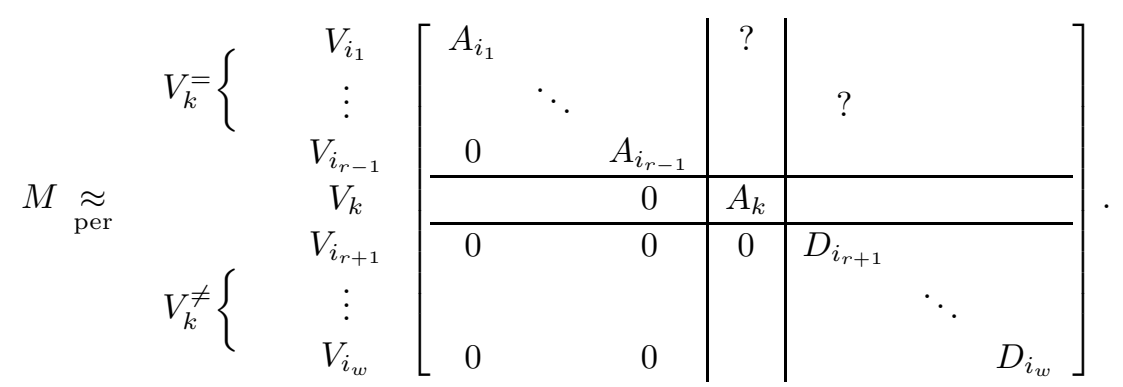

We may repeat this by selecting a first critical node in the matrix $D$, while keeping the same increasing ordering, etc. Labeling the nodes according to the critical nodes, we end up with

$$
M \underset{\text { per }}{\approx} F^{\prime}=\left[\begin{array}{cccc|c}
M_{1} & & & ? & \\
0 & M_{2} & & & \\
& & \ddots & & \\
& & & M_{s} & ? \\
\hline 0 & & & & T
\end{array}\right]
$$

with $\rho(T)<1$, where

$$
M_{i}=\begin{array}{c|ccc|c}
V_{1}^{(i)} \\
\vdots \\
V_{r_{i}-1}^{(i)} \\
V_{r_{i}}^{(i)}
\end{array}\left[\begin{array}{cccc}
A_{1}^{(i)} & & & ? \\
& \ddots & & \\
& & A_{r_{i}-1}^{(i)} & \\
\hline 0 & & & A_{r_{i}}^{(i)}
\end{array}\right]=\left[\begin{array}{cc}
U_{i} & V_{i} \\
0 & A_{r_{i}}
\end{array}\right]
$$

is again block triangular for $i=1, \ldots, s$. In addition:

(i) each corner block $A_{r_{i}}^{(i)}=A_{r_{i}}$ in each $M_{i}, i=1, \ldots, s$, corresponds to a critical node $V_{r_{i}}, i=1, \ldots, s$;

(ii) each of the upper nodes $V_{j}^{(i)}$ with $j=1, \ldots, r_{i}-1$, has access to corner node $V_{r_{i}}^{(i)}$

(iii) $T$ is block triangular but contains no critical nodes. 
Consider the form

$$
F^{\prime}=\left[\begin{array}{cc|cc|cc|c|cc||c}
U_{1} & V_{1} & ? & & & & & & \\
0 & A_{r_{1}} & X_{11} & X_{12} & X_{21} & X_{22} & \cdots & X_{s 1} & X_{s 2} & \\
\hline & 0 & U_{2} & V_{2} & & & & & & \\
& 0 & A_{r_{2}} & Y_{21} & Y_{22} & \cdots & Y_{s 1} & Y_{s 2} & \\
\hline & & & & U_{3} & V_{3} & & & & \\
& & & 0 & A_{r_{3}} & \cdots & Z_{s 1} & S_{s 2} & ? \\
\hline & & & & & \ddots & & & \\
\hline & & & & & & U_{s} & V_{s} & ? \\
& & & & & & & 0 & A_{r_{s}} & ? \\
\hline \hline 0 & & & & & & & & T
\end{array}\right]
$$

where the critical blocks $A_{r_{i}}$ correspond to the critical nodes $V_{r_{i}}$.

Since the singular node $V_{r_{1}}$ has no access to the other singular nodes we can conclude that $X_{i 1}=0$ and $X_{i 2}=0$. Likewise, all $Y_{i j}=0, Z_{i j}=0, \ldots$ That is, the block rows containing the $A_{r_{i}}$ do not contain any other nonzero blocks, except for the last block column. This means that we may permute the singular nodes, by first taking the odd block rows, except the last one, and then every odd block column, except the last one, followed by taking every even block row and then every even block column. This leads to

THEOREM 4.1. A nonnegative matrix $M$ is convergent (respectively, power bounded) if and only if it is permutation similar to a matrix of the form

$$
M^{\prime}=\left[\begin{array}{cc|c}
A & B & C \\
0 & D & E \\
\hline 0 & 0 & T
\end{array}\right]
$$

where

(i) $A$ and $T$ are block upper triangular;

(ii) the diagonal blocks $A_{i i}$ and $T_{j j}$ are irreducible or $0_{1 \times 1}$;

(iii) $\rho\left(A_{i i}\right)<1, \rho\left(T_{i i}\right)<1$;

(iv) $D=\operatorname{diag}\left(D_{1}, \ldots, D_{q}\right)$, in which $D_{i}=A_{r_{i}}$ is irreducible, $\rho\left(D_{i}\right)=1$ and $D_{i}$ is convergent (respectively, power bounded);

(v) $B, C$ and $E$ are arbitrary.

Since $D \mathbf{u}=1 \cdot \mathbf{u}$, for some $\mathbf{u}>\mathbf{0}$, it is easily seen that, for all $N$, the convolution product $\sum_{k=0}^{N-1} A^{N-k-1} B D^{k}$ is bounded exactly when $\sum_{k=0}^{N-1} A^{N-k-1} B \mathbf{u}$ is bounded. This suffices to show that if $\rho(A)<1$, then the matrix $M^{\prime}$ in (4.1) is also power bounded. $\square$ 
Given a nonnegative matrix $A$, let $\pi(A)$ be the number of eigenvalues of $A$ (including multiplicities) on the Perron circle $\{\lambda:|\lambda|=\rho(A)\}$. We now may state

Lemma 4.2. The following are equivalent for $A \geq 0$ :

(i) $A^{N}>0$ for some $N$.

(ii) $A$ is irreducible and $\pi(A)=1$.

(iii) $A$ is irreducible and $(A / \rho)^{N}$ converges to some $L>0$.

In which case, $\lim _{N}(A / \rho)^{N}=\frac{\mathbf{u v}^{T}}{\mathbf{v}^{T} \mathbf{u}}$, where $A \mathbf{u}=\rho \mathbf{u}$ and $A^{T} \mathbf{v}=\rho \mathbf{v}$.

Proof. This follows from [8, Theorem 8.5.1] and from [8, Theorem 8.5.2].

For the irreducible central matrices $D_{i}$ as in (4.1), we need the following result.

Lemma 4.3. Let $D$ be nonnegative irreducible with $\rho(D)=1$. Then

(a) The following are equivalent:

(i) $D$ is convergent.

(ii) $D^{N}>0$ for some $N$.

(iii) $\pi(D)=1$.

In either case, $D^{k} \rightarrow \frac{\mathbf{u v}^{T}}{\mathbf{v}^{T} \mathbf{u}}$ for some positive $\mathbf{u}, \mathbf{v}$.

(b) If $D$ is irreducible then $D$ is $P B$ if and only if $U^{-1} D U=P$, for some irreducible stochastic matrix $P$, and $U=d g\left(u_{1}, \ldots, u_{n}\right)$ with $u_{i}>0$

Proof. (a) This follows from Lemma 4.2.

(b) By the Perron-Frobenius Theorem $D \mathbf{u}=1 \cdot \mathbf{u}$, where $\mathbf{u}>\mathbf{0}$. Since $D \mathbf{e}=\mathbf{u}$, where $\mathbf{e}=[1,1, \ldots, 1]^{T}$, we now obtain $U^{-1} D U=P$ with $P \mathbf{e}=1 \cdot \mathbf{e}$, as desired.

Example 1.1 shows that Theorem 4.1 is best possible.

We note in passing that for a nonnegative $\mathrm{PB}$ matrix, $a_{i i} \leq \rho(A) \leq 1$.

If $M$ is periodic, then there is a first power of $M$, say $M^{b+h}$, that is equal to an earlier power $M^{b}$, and thus,

$$
M^{b}=M^{b+h} .
$$

This means that $x^{b}\left(x^{h}-1\right)$ is the minimal polynomial for $M$ with $\rho(M)=1$ and $h$ is the Boolean period of $M$. Consequently, the matrices $A$ and $T$ in the canonical form of equation (4.1) must be nilpotent and $D^{b}=D^{b+h}$. Let us next focus on one of the direct summands in $D=\operatorname{diag}\left(D_{1}, \ldots, D_{q}\right)$.

Consider a nonnegative, irreducible matrix $D$ such that $\rho(D)=1$ and $D^{b}=D^{b+h}$. It is known [5, pp 53-54], 2, Theorem 2.20] that $D$ is permutation similar to its cyclic 
form

$$
D \underset{\text { per }}{\approx} E=\left[\begin{array}{ccccc}
0 & X_{1} & 0 & & 0 \\
0 & 0 & X_{2} & & \\
& & \ddots & & \\
0 & 0 & 0 & & X_{f-1} \\
& & & \ddots & \\
X_{f} & 0 & & & 0
\end{array}\right],
$$

where the diagonal blocks are square and $f$ will be referred to as the Perron period of $D$.

It further follows that $E^{f}=\operatorname{diag}\left(B_{0}, B_{1}, \ldots, B_{f-1}\right)$, where each $B_{i}$ is such that $B_{i}^{\varepsilon_{i}}>0$, for some $\varepsilon_{i}$, and hence, $\pi\left(B_{i}\right)=1$. If we set $\varepsilon=\max \left\{\varepsilon_{i}\right\}$, then $E^{f \varepsilon}$ is a direct sum of positive matrices. In addition $\rho\left(B_{i}\right)=\rho\left(B_{0}\right)=[\rho(E)]^{f}=1$. This means that

$$
\Delta_{D}(x)=x^{n_{0}}\left(x^{f}-1\right) \prod_{2}^{q}\left(x^{f}-\gamma_{k}\right)^{n_{k}},
$$

where $n_{0}$ is the algebraic multiplicity of the eigenvalue 0 and $0<\left|\gamma_{k}\right|<1$.

On the other hand, if $D^{b}=D^{b+h}$ then the minimal polynomial of $D$ is given by $\psi(x)=x^{b}\left(x^{h}-1\right)$, which has $h$ distinct roots $\lambda_{i}$ on the unit circle. This means that $\Delta_{D}(x)$ takes the form

$$
\Delta_{D}(x)=x^{n_{0}}\left(x-\lambda_{1}\right)^{k_{1}} \cdots\left(x-\lambda_{h}\right)^{k_{h}} .
$$

Identities (4.2) and (4.3) show that the $\gamma_{i}$ must be absent and that all $k_{i}=1$. It then follows that $f=h$, i.e., the Perron period and the Boolean period are equal.

Next, we observe that $E$ is also periodic with $E^{b}=E^{b+h s}$ for $s=1,2, \ldots$ Two cases can occur.

Case (I). If $b \leq h$ then multiplying through by $E^{h-b}$ shows that $E^{h}=E^{2 h}$. This means that $E^{h \varepsilon}=E^{2 h \varepsilon}=\operatorname{diag}\left(B_{0}^{\varepsilon}, \ldots, B_{h-1}^{\varepsilon}\right)$, in which each $B_{i}^{\varepsilon}$ is a positive idempotent.

Case (II). When $b>h$, we write $b=q h+r$, where $0 \leq r<h$. Then $E^{b}=$ $\left(E^{h}\right)^{q} \cdot E^{r}=\left(E^{h}\right)^{q+1} \cdot E^{r}$. Post-multiplying by $E^{h-r}$ then shows that $\left(E^{h}\right)^{q+1}=$ $\left(E^{h}\right)^{q+2}=\left(E^{h}\right)^{q+3}=\cdots$, and thus, $\left(E^{h \varepsilon}\right)^{q+1}=\left(E^{h \varepsilon}\right)^{2(q+2)}$. This means that we again have a block diagonal idempotent matrix with positive diagonal blocks. Thus, we have the following result:

COROllary 4.4. A matrix is periodic, nonnegative irreducible with spectral radius one if and only if it has a power that is permutation similar to a direct sum of positive idempotents. 
We recall in passing that

LEMMA 4.5. A positive idempotent matrix must have rank one.

Proof. Let $E=E^{2}$ be positive $n \times n$. Then the Jordan form of $E$ is of the form $I_{r} \oplus 0_{n-r}$. Since $E>0$, it follows from the Perron-Frobenius theorem that $r=1$.

A positive periodic matrix must have a Boolean period of one and a minimal polynomial of the form $x^{b}(x-1)$, but it need not be idempotent. For example, consider any positive stochastic matrix with trace one and rank two.

Example 4.6. The matrix $A=\left[\begin{array}{ccc}1 / 6 & 1 / 3 & 1 / 3 \\ 1 / 6 & 1 / 3 & 1 / 2 \\ 1 / 4 & 1 / 4 & 1 / 2\end{array}\right]$ is positive, periodic, but not idempotent.

Let us now turn to a special nonnegative PB case, in which we capitalize on the fact that the two periods are equal.

\section{Entries in nonnegative $\mathrm{PB}$ matrices.}

5.1. Basics. We begin by listing some of the pertinent facts about a nonnegative $\operatorname{matrix} M=\left[m_{i j}\right]$.

(1) $\rho(M)$ is an eigenvalue of $M$ and there exists a nonnegative eigenvector associated with $\rho(M)$.

(2) $m_{i i} \leq \rho(M)$.

(3) If $M=\left[\begin{array}{ll}A & C \\ B & D\end{array}\right] \geq 0$ and $M^{N}=\left[\begin{array}{ll}A_{N} & C_{N} \\ B_{N} & D_{N}\end{array}\right]$ then $A_{N} \geq A^{N}$.

(4) $\left(M^{N}\right)_{i i} \geq\left(m_{i i}\right)^{N}$ for $N=1,2, \ldots$

(5) $\sum_{i=1}^{n} m_{i i}^{N} \leq \operatorname{Tr}\left(M^{N}\right)$ for $N=1,2, \ldots$

Next, consider a nonnegative power bounded (nnPB) matrix $M$. First, some general results.

\section{LEMMA 5.1.}

(i) If $A, B \geq 0$ and $A+B$ is $P B$, then so are $A$ and $B$.

(ii) If $M=\left[\begin{array}{ll}A & C \\ B & D\end{array}\right]$ is nnPB, then so are $A$ and $D$.

(iii) If $M$ is nnPB then so are all principal submatrices of $M$.

Proof. (i) Clearly, $(A+B)^{N} \geq A^{N}$. 
(ii) If $M=\left[\begin{array}{ll}A & 0 \\ B & 0\end{array}\right]+\left[\begin{array}{ll}0 & C \\ 0 & D\end{array}\right]$ is $\mathrm{PB}$, then (by part (i)), so are its two components. It is clear that $\left[\begin{array}{ll}A & 0 \\ B & 0\end{array}\right]^{N}=\left[\begin{array}{cc}A^{N} & 0 \\ ? & 0\end{array}\right]$, which guarantees that $A$ is PB.

(iii) Clear by permuting rows and columns.

Let us now turn to the diagonal entries of a nnPB matrix.

5.2. Diagonal 3ntries of a nonnegative PB matrix. Let $M=\left[m_{i j}\right]$ be a nnPB matrix.

Using Theorem 2.1 and the nonnegativity, we may conclude that

$$
m_{i i} \leq \rho(M) \leq 1
$$

This also follows from the fact that $\left(m_{i i}\right)^{N} \leq\left(M^{N}\right)_{i i}$, which is bounded.

Our aim is to use the diagonal entries of $M$ to investigate power boundedness and at the same time to relate the three concepts of $\mathrm{PB}$, focal power, and convergence.

The two main cases to be considered are when some $m_{i i}=1$ or when all $m_{i i}<1$. Only in the former case does focal power play a role. In the latter case there is little we can say about the form of $M$, as seen from the example $M=\left[\begin{array}{ll}.8 & .2 \\ .2 & .8\end{array}\right]$, which has eigenvalues $\{1,0.6\}$.

We next have

LemmA 5.2. If $M$ is nnPB, then, for all natural numbers $k, N$,

(i) $m_{i i}^{k} \leq m_{i i}$

(ii) $\sum_{i=1}^{n} m_{i i}^{k} \leq \operatorname{Tr}(M)$, and

(iii) $m_{i i}=1 \Rightarrow\left(M^{N}\right)_{i i}=1$.

Proof. (i) and (ii) follow from the inequalities (5.1).

(iii). $M^{k}$ is also PB with $\left(M^{k}\right)_{i i} \leq 1$. Hence, $1=\left(m_{i i}\right)^{N} \leq\left(M^{N}\right)_{i i} \leq 1$.

The matrix $M=\left[\begin{array}{ll}0 & 1 \\ 1 & 0\end{array}\right]$ has the property that $\left(M^{2}\right)_{i i}=1$ while $m_{i i}=0$; thus, we cannot reverse the conditions of (iii).

We now introduce the parameters $u_{k}=\#\left\{i:\left(M^{k}\right)_{i i}=1\right\}$ and $t_{k}=\operatorname{Tr}\left(M^{k}\right)$. 
Then it easily folows that

$$
u_{1} \leq u_{k} \leq t_{k} \leq n
$$

We note that the sequence $\left\{t_{k}\right\}$ need not be increasing, as seen from $M=$ $\left[\begin{array}{ll}0 & 1 \\ 1 & 0\end{array}\right]$. Also, if $M$ is not nilpotent, then some $t_{k}$ must be nonzero.

Suppose that $v=\max \left\{u_{k}\right\}$ and $w=\max \left\{t_{k}\right\}$ and let $\Delta=\left\{i: u_{i}=v\right\}$ and $\Gamma=\left\{i: \operatorname{Tr}\left(A^{i}\right)=w\right\}$. Clearly, if $u_{r}=v$ then $u_{r^{k}}=v$ for all $k \geq 1$.

\section{LEMMA 5.3.}

(i) If $M=I+X$ is nnPB, with $X \geq 0$, then $X=0$.

(ii) If $M=\left[\begin{array}{cc}I+X & ? \\ ? & ?\end{array}\right]$ is nnPB, with $X \geq 0$, then $X=0$.

(iii) If $M=\left[\begin{array}{ll}I & C \\ B & D\end{array}\right]$ is nnPB, then $M$ is (1,1) focussed.

Proof. (i) $M^{N}=(I+X)^{N} \geq I+N X$. For $M$ to be power bounded, we must have $X=0$.

(ii) Since $I+X$ is nnPB, this follows from part (i).

(iii) $M^{N}$ has the form $\left[\begin{array}{cc}I+X & C_{N} \\ B_{N} & D_{N}\end{array}\right] \geq 0$. By part (ii) $X$ must vanish.

Corollary 5.4. If $M$ is nnPB and all $m_{i i}=1$, then $M=I$.

5.3. Unit diagonal entries. Suppose now that $M$ is nnPB and has some diagonal elements $m_{i i}=1$.

Applying Lemma 5.3 (ii), we may permute the rows and columns of $M$ so that

$$
M \underset{\text { per }}{\approx} M^{\prime}=\left[\begin{array}{cc}
I_{u_{1}} & C \\
B & D
\end{array}\right]
$$

where $D$ is also nnPB and all $d_{i i}<1$. From Lemma 5.3 (iii) we know that $M^{\prime}$ is $(1,1)$ focussed and that $M^{\prime N}=\left[\begin{array}{cc}I_{u_{1}} & C_{N} \\ B_{N} & D_{N}\end{array}\right]$. Moreover, we also know, using [7, Theorem 1.1], that, for all $N$,

$$
\begin{array}{lrl}
\text { (1) } C D^{N} B & =0, \\
\text { (2) } B_{N} & =G_{N} B=\left(I+D+\cdots+D^{N-1}\right) B \text { is bounded, } \\
\text { (3) } & C_{N}=C G_{N}=C\left(I+D+\cdots+D^{N-1}\right) \text { is bounded, } \\
\text { (4) } & D_{N}=D^{N}+X_{N} \text { where } X_{N}=\sum_{p+q \leq N-2} D^{p} B C D^{q} .
\end{array}
$$


For convenience we do not distinguish between $M$ and $M^{\prime}$.

It should be noted that:

(i) Condition (1) implies that $C B_{N}=0=C_{N} B$.

(ii) $X_{N}^{2}=0$ (by (1)).

(iii) $X_{N}$ is increasing with $N$.

(iv) $X_{N} \leq G_{N} B C G_{N}$, which is bounded by (2) in (5.2). Therefore, $X_{N}$ converges.

(v) Taking traces shows that $\operatorname{Tr}\left(D^{p} B C D^{q}\right)=\operatorname{Tr}\left(C D^{p+q} B\right)=0$, and hence, that

$$
\operatorname{Tr}\left(D_{r}\right)=\operatorname{Tr}\left(D^{r}\right), r=1,2, \ldots
$$

We next need

LEMma 5.5. $G_{N}(F) B$ is bounded for all $N$ if and only if $F^{N} B$ is bounded and $Z_{1}^{0}(F) B=0$.

Proof. If $G_{N}(F) B$ is bounded for all $N$ then so is $(I-F) G_{N} B=\left(I-F^{N}\right) B$, and hence, $F^{N} B$ is bounded as well. Next we post-multiply (2.1) by $B$ giving

$$
G_{N}(F) B=(I-F)^{d}\left(I-F^{N}\right) B+\sum_{j=0}^{m_{1}-1}\left(\begin{array}{c}
N \\
j+1
\end{array}\right) Z_{1}^{j} B
$$

in which the left hand side and the first term on the right hand side are bounded. Now pre-multiply by $Z_{1}^{m_{1}-1}$. This leaves us the bounded term $N Z_{1}^{m_{1}-1} B$. This cannot be, unless $Z_{1}^{m_{1}-1} B=0$. Next we multiply by $Z_{1}^{m_{1}-2}$ etc., implying eventually that $Z_{1}^{0}(F) B=0$.

Conversely, if $Z_{1}^{0}(F) B=0$ then the second term on the right hand side of (5.3) vanishes, while the first term is bounded because $F^{N} B$ is bounded.

Let us now apply the previous Lemma to the block matrix $M$.

Proposition 5.6. The matrix $M=\left[\begin{array}{cc}I_{u_{1}} & C \\ B & D\end{array}\right] \geq 0$ is $P B$ if and only if $M$ is $(1,1)$ focussed, $D$ is $P B$ and $Z_{1}^{0} B=0=C Z_{1}^{0}$, where $Z_{1}^{0}=I-(I-D)(I-D)^{\#}$.

Proof. We have seen that if $M$ is PB then necessarily, $D$ is PB, $M$ is $(1,1)$ focussed, and $G_{N} B$ and $C G_{N}$ are bounded, for all $N$. The latter ensure that $Z_{1}^{0} B=0=C Z_{1}^{0}$ by (5.3).

Conversely, if $M$ is $(1,1)$ focussed then $M^{N}=\left[\begin{array}{cc}I_{u_{1}} & C_{N} \\ B_{N} & D_{N}\end{array}\right]$ in which $B_{N}$ and $C_{N}$ are bounded by Lemma [5.5, and $D_{N}=D^{N}+X_{N}$ is bounded because $D$ is PB and (iii) $X_{N} \leq G_{N} B C G_{N}$ is also bounded, for all $N$. 
A special subcase is when $\rho(D)<1$, which includes the case where $D$ is nilpotent and $\rho(D)=0$. In this case $D^{N} \rightarrow 0, B_{N} \rightarrow(I-D)^{-1} B$, and $C_{N} \rightarrow C(I-D)^{-1}$. In addition, $D_{N}=D^{N}+X_{N}$ in which $D^{N} \longrightarrow 0$ and $X_{N}=\sum_{p+q \leq N-2} D^{p} B C D^{q}$ is monotone increasing and is bounded above by $(I-D)^{-1} B C(I-D)^{-1}$ and as such, converges.

This leads to

Corollary 5.7. If $M=\left[\begin{array}{ll}I & C \\ B & D\end{array}\right]$ is (1,1) focussed, $Z_{1}^{0}(D) B=0=C Z_{1}^{0}(D)$, and $\rho(D)<1$, then $M^{N}$ converges to $Z_{1}^{0}(M)$.

5.4. No unit diagonal entries. Let $M$ be $\mathrm{PB}$ with all $m_{i i}<1$, as was the case with the matrix $D$ in the previous setting. It is not clear if a power of $M$ can have unit diagonal entries. We may or may not have $\rho(M)=1$. For example $M=\left[\begin{array}{ll}.5 & .1 \\ .1 & .5\end{array}\right]$ has eigenvalue of $\{.6, .4\}$, and thus, is zero convergent.

We may even have a zero diagonal, as seen from the example $M=\left[\begin{array}{ll}0 & c \\ b & 0\end{array}\right]$ with $|b c| \leq 1$. Indeed, $M^{2 k}=(b c)^{k} I$. This example also shows that for a nnPB matrix some of its entries may be larger than 1 .

Let us now turn to a special class of PB matrices where we keep the diagonal entries under control and can effectively employ the trace concept. This in turn allows us to bring the previous "diagonal" argument to a more transparent conclusion.

6. The semigroup case of nonnegative PB matrices. Consider a nonnegative PB matrix $M$ for which $\left(M^{k}\right)_{i i}=0$ or 1 (only), for all powers $k=1,2, \ldots$ Our aim is to show that such a matrix must be periodic, and that a power of $M$ is permutation similar to an idempotent matrix.

If $M$ is nilpotent, then $M$ is periodic and the idempotent is zero. So let us assume that $M$ is nilpotent in what follows.

First we need some simple observations.

If $M$ is $\mathrm{nnPB}$ and $m_{i i}=0$ or 1 , then $m_{i i}^{k}=m_{i i}$ and $u_{k}=t_{k}$ for all $k=1,2, \ldots$, and hence, we can combine the two parts of Lemma 5.2 in

$$
\operatorname{Tr}\left(M^{k}\right) \geq \sum_{i=1}^{n} m_{i i}^{k}=\sum_{i=1}^{n} m_{i i}=\operatorname{Tr}(M) \text { for } k=1,2, \ldots,
$$

i.e., $t_{1} \leq t_{k}$ for all $k$. In general, for a $\mathrm{nnPB}$ matrix, we saw that $0 \leq m_{i i} \leq 1$ and hence the assumption that $m_{i i}$ can only equal 0 or 1 is an extremal condition. 
Because of the assumption that $\left(M^{k}\right)_{i i}=1$ or $\left(M^{k}\right)_{i i}=0$, we see that for every $k=0,1, \ldots$

$$
M^{k} \underset{\text { per }}{\approx}\left[\begin{array}{cc}
I_{t_{k}}+X_{k} & ? \\
? & N_{k}
\end{array}\right]
$$

where $t_{k}=\operatorname{Tr}\left(M^{k}\right), X_{k} \geq 0,\left(X_{k}\right)_{i i}=0$ and $\left(N_{k}\right)_{i i}=0$. Lemma 5.3 ensures that $X_{k}=0$ and we may state that for all $k=0,1, \ldots$

$$
M^{k} \underset{\text { per }}{\approx}\left[\begin{array}{cc}
I_{t_{k}} & ? \\
? & N_{k}
\end{array}\right]
$$

with $\left(N_{k}\right)_{i i}=0$. Using the same argument, we see that for $r=1,2, \ldots$

$$
\left(M^{k}\right)^{r} \underset{\text { per }}{\approx}\left[\begin{array}{cc}
I_{t_{k}} & ? \\
? & ?
\end{array}\right],
$$

which has $\operatorname{Tr}\left(M^{k r}\right) \geq t_{k}$ and has at least $t_{k}$ ones on its diagonal.

Next we return to the case where $k=1, M \underset{\text { per }}{\approx} M^{\prime}=\left[\begin{array}{ll}I_{t} & C \\ B & D\end{array}\right], t=t_{1}=$ $\operatorname{Tr}(M), B=B_{1}, C=C_{1}$, and $(D)_{i i}=0$. Then from (6.2), we may conclude that

$$
M^{r} \underset{\text { per }}{\approx}\left(M^{\prime}\right)^{r}=\left[\begin{array}{cc}
I_{t} & C_{r} \\
B_{r} & D_{r}
\end{array}\right]
$$

which is $(1,1)$ focussed and has $\operatorname{Tr}\left(M^{r}\right) \geq t$.

In addition we know that

(i) $\operatorname{Tr}\left(D^{r}\right)=\operatorname{Tr}\left(D_{r}\right)=\operatorname{Tr}\left(M^{r}\right)-\operatorname{Tr}(M) \leq n-t$,

(ii) $\operatorname{Tr}\left(D^{r}\right)$ may oscillate, as seen from $D=\left[\begin{array}{ll}0 & 1 \\ 1 & 0\end{array}\right]$ with $D^{2}=I$.

Since $M$ is nonnegative non-nilpotent, there exists a power of $M$, say $M^{u}$, with $\operatorname{Tr}\left(M^{u}\right)>0$ (recall that $M$ is nilpotent if and only if all powers have zero trace!).

We now focus on

(a) $w=\max \left\{\operatorname{Tr}\left(M^{k}\right) ; k \in \mathbb{N}\right\}=\max \left\{t_{k}\right\}$ and (b) $\Gamma=\left\{i ; \operatorname{Tr}\left(M^{i}\right)=w\right\}$.

The set $\Gamma$ must be nonempty. Now suppose that $r \in \Gamma$, i.e., $\operatorname{Tr}\left(M^{r}\right)=w$. By (6.1) applied to $M^{r}$, we see that

$$
w=\operatorname{Tr}\left(M^{r}\right) \leq \operatorname{Tr}\left(M^{r s}\right) \leq w \text { for all } s,
$$

and thus, $r s \in \Gamma$ for $s=1,2, \ldots$ This means that

$$
\Gamma+\Gamma \subseteq \Gamma
$$


Thus, $\Gamma$ is an additive subsemigroup of $\mathbb{N}$.

$$
\begin{gathered}
\text { Next, let } M^{r} \underset{\text { per }}{\approx} Y=\left[\begin{array}{cc}
I_{w} & L \\
K & \eta
\end{array}\right] \text {, where }(\eta)_{i i}=0 \text {. By (6.3) we see that } \\
Y^{i}=\left[\begin{array}{ll}
I_{w} & L_{i} \\
K_{i} & \eta_{i}
\end{array}\right]
\end{gathered}
$$

so that $\operatorname{Tr}\left(Y^{i}\right)=\operatorname{Tr}\left(M^{r i}\right)=w+\operatorname{Tr}\left(\eta_{i}\right)$ for all $i=1,2, \ldots$ By the maximality of $w$ we conclude that $\operatorname{Tr}\left(\eta_{i}\right)=0$ for all $i=1,2, \ldots$ In addition, we see from (5.2) that

$$
\begin{aligned}
& \text { (i) } \quad L \eta^{i} K=0 \text { for all } i=0,1, \ldots, \\
& \text { (ii) } K_{i}=\left(I+\eta+\cdots+\eta^{i-1}\right) K \text {, } \\
& \text { (iii) } L_{i}=L\left(I+\eta+\eta^{2}+\cdots+\eta^{i-1}\right) \text {, }
\end{aligned}
$$

as well as

$$
\eta_{i}=\eta^{i}+\sum_{p+q \leq i-2} \eta^{p} K L \eta^{q}
$$

The identity (6.7) implies that $0=\operatorname{Tr}\left(\eta_{i}\right)=\operatorname{Tr}\left(\eta^{i}\right)$ for $i=0,1, \ldots$ That is, $\eta$ is nilpotent, say of index $i n(\eta)=s$.

We now claim that matrices of the form (6.5) are in fact periodic. Indeed, it is clear that $K_{i} \rightarrow(I-\eta)^{-1} K$ and $L_{i} \rightarrow L(I-\eta)^{-1}$ so both sequences are bounded. Moreover, from (6.7), we see that if $i=2 s+t+2$, then we may split the sum into

$$
\sum_{p+q \leq 2 s} \eta^{p} K L \eta^{q}+\sum_{2 s<p+q \leq 2 s+t} \eta^{p} K L \eta^{q} .
$$

In the second summand, either $p \geq s$ or $q \geq s$, and hence, this term vanishes. Thus, $\eta_{i}$ reduces to just a finite (bounded) sum. Let us now take $i=s$ and partition $Y^{s}$ as

$$
Y^{s}=\left[\begin{array}{cc}
I_{w} & L(I-\eta)^{-1} \\
(I-\eta)^{-1} K & \eta_{s}
\end{array}\right]
$$

where $\eta_{s}=\sum_{p+q \leq s-2} \eta^{p} K L \eta^{q}$. Using the fact that $L \eta^{j} K=0$ for all $j$, we see that $\left(\eta_{s}\right)^{2}=0$ in addition to $L(I-\eta)^{-1} \eta_{s}=0$. Likewise if we use (6.6) then it follows that $\eta_{s}(I-\eta)^{-1} K=0$ and $L(I-\eta)^{-2} K=0$. We now apply the following trivial result

Lemma 6.1. If $G=\left[\begin{array}{ll}I & Q \\ P & R\end{array}\right]$ with $R^{2}=0, Q P=0, R P=0$ and $Q R=0$, then $G^{2}$ is idempotent.

It now follows that for $s=i n(\eta), Y^{2 s}$ is idempotent and hence is periodic. We have thus shown that if $M$ is nonnegative power bounded, with $\left(M^{k}\right)_{i i}=0,1$, then $M^{2 r s}$ is idempotent. 
Conversely, if $M^{m}$ is idempotent then it is trivially power bounded. Moreover, it follows that $M$ is also PB. Indeed, for any $k \in \mathbb{N}$, let $k=t m+\alpha$ with $0 \leq \alpha<r$. Further let $\left|\left(M^{t m}\right)_{i j}\right| \leq K_{1}$ for all $i, j, t$, and let $K_{2}=\max \left\{\sum_{j=1}^{n}\left|\left(M^{\alpha}\right)_{p j}\right| ; j=\right.$ $1, \ldots, m, \alpha=0,1, \ldots, m-1\}$. Then $\left|\left(M^{k}\right)_{i j}\right| \leq K_{1} K_{2}$ for all $i, j, k$.

\section{Remarks.}

(a) To accommodate "large" entries besides (possible) zero entries, in a nnPB matrix $M$, it is not surprising that $M$ has to be periodic.

(b) One instance where our matrices do have 0-1 power diagonals is furnished when the entries in $M$ come from a sub-semigroup $S^{*}$ of $\mathbb{R}^{+}$for which the following implication holds [14]:

(i) $S^{*}+S^{*} \subseteq S^{*}$, (ii) $S^{*} \cdot S^{*} \subseteq S^{*}$, (iii) $0,1 \in S^{*}$, and (iv) $x \in S^{*}, x \neq 0 \Rightarrow$ $x \geq 1$.

(c) The eigenvalues of a periodic matrix are either zero or lie on the unit circle. On the other hand, the eigenvalues of an irreducible nonnegative matrix lie symmetrically around the unit circle. This means that the index of primitivity $\pi(A)$ equals the periodicity.

(d) Can the zero-one structure of the diagonals of the powers be used to obtain a canonical form for PB matrices?

Acknowledgement. This research was financed by FEDER Funds through "Programa Operacional Factores de Competitividade - COMPETE" and by Portuguese Funds through FCT - "Fundação para a Ciência e a Tecnologia", within the project PEst-C/MAT/UI0013/2011.

The authors wish to thank the referee for numerous constructive suggestions, and for providing an alternative proof for Lemma 4.5 .

\section{REFERENCES}

[1] A. Ben-Israel and T.N.E. Greville. Generalized Inverses: Theory and Applications. John Wiley and Sons, New York, 1974.

[2] A. Berman and R.J. Plemmons. Nonnegative Matrices in the Mathematical Sciences. Society for Industrial and Applied Mathematics (SIAM), Philadelphia, 1994.

[3] X. Chen and R.E. Hartwig. The Picard iteration and its application. Linear Multilinear Algebra, 54(5):329-341, 2006.

[4] D.T. Finkbeiner. Introduction to Matrices and Linear Transformations, second edition. W.H. Freeman and Co., San Francisco, 1966.

[5] F.R. Gantmacher. The Theory of Matrices vol. 2. Translated from the Russian by K.A. Hirsch. Reprint of the 1959 translation. AMS Chelsea Publishing, Providence, RI, 1998.

[6] R.E. Hartwig. A note on periodic matrices. Indust. Math., 27:51-55, 1977.

[7] R.E. Hartwig and J. Maroulas. Focal power. Electron. J. Linear Algebra, 13:56-71, 2005. 
[8] R. Horn and C.R. Johnson. Matrix Analysis. Cambridge University Press, Cambridge, 1990.

[9] A. Ostrowski. Über Normen von Matrizen. Math. Z., 63:2-18, 1955.

[10] M. Neumann and R.J. Plemmons. Convergent nonnegative matrices and iterative methods for consistent linear systems. Numer. Math., 31:265-279, 1978/79.

[11] M. Neumann and R.J. Plemmons. Generalized inverse-positivity and splittings of $M$-matrices. Linear Algebra Appl., 23:21-35, 1979.

[12] U. Rothblum. A rank characterization of the number of final classes of a nonnegative matrix. Linear Algebra Appl., 23:65-68, 1979.

[13] U. Rothblum. Expansions of sums of matrix powers. SIAM Rev., 23:143-164, 1981.

[14] S. Yang and R. Zhang. Green's relations in the matrix semigroup $M_{n}(\mathcal{S})$. Linear Algebra Appl., 222:63-76, 1995. 